\title{
Diagnóstico do Comportamento dos Aprendizes na Educação a Distância com Base no Estilo de Aprendizagem
}

\author{
Leonardo Heidrich $^{1}$, Jorge Barbosa ${ }^{1}$, Sandro Rigo ${ }^{1}$, \\ Wagner Cambruzzi ${ }^{1}$, Giovane Ribeiro ${ }^{1}$ \\ ${ }^{1}$ Universidade do Vale do Rio dos Sinos (UNISINOS) \\ Av. Unisinos, 950 - São Leopoldo - RS - Brasil \\ \{leonardoheidrich, giovaned.ribeiro\}@gmail.com, \\ \{jbarbosa,rigo\}@unisinos.br, wagner@cambruzzi.com.br
}

\begin{abstract}
The data generated by the use of systems such as Virtual Learning Environments contain information that can be used to support teachers in decision making, which may be required even before obtaining historical data. The learner Learning Style can complement this data covering the gap through the intersection with the historical behavior patterns. Thus, this work aims to propose an evaluation of the potential use of Learning Style in the early learner behavior diagnosis in the distance learning to support decision making. The results, of the experiment focused on the dropout behavior, suggest that the learning style can be used in the learner behavior prediction.
\end{abstract}

Resumo. Os dados gerados pela utilização de sistemas como Ambientes Virtuais de Aprendizagem contêm informações que podem ser utilizadas para auxiliar os professores na tomada de decisão, que em alguns casos, pode ser requerida antes mesmo de se obter dados históricos. $O$ Estilo de Aprendizagem pode complementar esses dados através do cruzamento com os padrões de comportamento histórico. Neste sentido, este trabalho propõe um modelo de utilização do Estilo de Aprendizagem no diagnóstico antecipado do comportamento de aprendizes na modalidade de Educação a Distância para o suporte à tomada de decisão. Os resultados alcançados em um experimento que focou na evasão sugerem que o Estilo de Aprendizagem pode ser usado para antecipação desse comportamento.

\section{Introdução}

Aprendizes possuem preferências distintas e aprendem de maneiras diferentes [Felder and Silverman 1988]. Conhecer os aprendizes individualmente se faz necessário para que o ensino seja adequado às suas preferências. Na Educação a Distância (EaD) esse conhecimento pode ser ainda mais crítico [Moore 1993]. Nesse sentido, os dados gerados pela utilização de sistemas como Enterprise Resource Planning (ERP), Learning Management System (LMS) e Ambiente Virtual de Aprendizagem (AVA) podem conter informações valiosas que podem ser utilizadas para auxiliar professores no suporte à tomada de decisões através do diagnóstico dos aprendizes [Siemens and Long 2011].

No entanto, os dados destes sistemas necessitam ser coletados e processados para obter-se as informações relevantes sobre os aprendizes. Neste sentido, os dados históricos dos aprendizes podem ser organizados segundo o conceito de Trilha 
[Silva et al. 2010]. A Trilha de Aprendizagem (TA) é o registro histórico de contextos [Dey et al. 2001] de aprendizagem visitados por um aprendiz. Essa informação, segundo [Johnson and Johnson 2010], permite identificar o comportamento do aprendiz durante o processo de aprendizagem. Porém, em determinadas situações deseja-se saber informações sobre os aprendizes antecipadamente sem ainda existir a disponibilidade de uma trilha de aprendizagem, por exemplo, no primeiro semestre de um curso de graduação. O Estilo de Aprendizagem (EA) representa as preferências de aprendizagem individuais e permanece estável com o passar do tempo [Peterson et al. 2009, Felder and Silverman 1988]. Logo o EA pode complementar os dados históricos e ser utilizado para tomada de decisão.

Na modalidade EaD, os AVAs são amplamente utilizados como ferramenta de suporte ao ensino e a Internet é o meio de comunicação mais utilizado para a troca de informações [Wu 2012]. Com o crescimento dos cursos de EaD, torna-se gradualmente mais evidente a possibilidade do uso de ferramentas de apoio ao professor, embasadas na utilização das diversas informações dos alunos. Nesta perspectiva, este artigo propõe um modelo para a avaliação da potencial utilização do Estilo de Aprendizagem no diagnóstico antecipado do comportamento dos aprendizes da modalidade $\mathrm{EaD}$ para o suporte à tomada de decisão.

O texto está estruturado da seguinte forma. A seção 2 apresenta os trabalhos relacionados. A seção 3 descreve o modelo proposto e a seção 4 a implementação de um protótipo. A seção 5 descreve o experimento focando evasão e discutindo os resultados alcançados. Por fim, a seção 6 apresenta as considerações finais e sugere trabalhos futuros.

\section{Trabalhos Relacionados}

O artigo [Wu 2012] propõe um sistema capaz de realizar o diagnóstico individual da situação atual do conhecimento dos aprendizes. O sistema tem como base o Learning Diagnosis Diagram (LDD), uma ferramenta de representação do conhecimento utilizada para fazer a auto adaptação do conteúdo a ser estudado pelo aprendiz. Essa ferramenta permite mapear sequencialmente todas as atividades realizadas pelos aprendizes.

O trabalho [Chen 2011] apresenta um sistema que implementa a técnica Pathfinder Network para prover ações remediais com base no conhecimento individual dos aprendizes para a modalidade de EaD.

Em [Chen and Bai 2009], os autores propõem um novo método para diagnosticar dificuldades dos aprendizes através do uso de Fuzzy Rules para sistemas de aprendizado adaptativo. $\mathrm{O}$ método busca inferir a probabilidade de um aprendiz em encontrar dificuldades em sua aprendizagem.

O artigo [Manghirmalani et al. 2011] propõe o uso de uma técnica chamada Learning Vector Quantization (LVQ) para diagnóstico e classificação de deficiências na aprendizagem como dislexia, disgrafia e discalculia.

No trabalho [Lee et al. 2009] é apresentado um sistema para diagnóstico de dificuldades dos aprendizes utilizando o algoritmo Apriori em um mapa Conceitual (Concept Map) orientado para a modalidade de EaD. Utilizando o Mapa Conceitual dos aprendizes, o Apriori cria Regras de Associação (Association Rules (AR)) com base na frequência de itens semelhantes. 
Tabela 1. Comparação entre Trabalhos Relacionados

\begin{tabular}{|c|c|c|c|c|c|c|c|}
\hline Trabalho & $\mathbf{D}$ & $\mathbf{T}$ & EaD & DH & EA & TA & $\mathbf{F}$ \\
\hline [Wu 2012] & $\checkmark$ & LDD & $\checkmark$ & $\checkmark$ & - & - & $\bar{\checkmark} \checkmark$ \\
\hline [Chen 2011] & $\checkmark$ & Pathfinder Network & $\checkmark$ & $\checkmark$ & - & - & - \\
\hline [Chen and Bai 2009] & $\checkmark$ & Fuzzy Rules & - & - & - & - & $\checkmark$ \\
\hline [Manghirmalani et al. 2011] & $\checkmark$ & LVQ & - & - & - & - & - \\
\hline [Lee et al. 2009] & $\checkmark$ & Apriori & $\checkmark$ & $\checkmark$ & - & - & $\checkmark$ \\
\hline [Chen et al. 2007a] & $\checkmark$ & Association Rules & $\checkmark$ & $\checkmark$ & - & - & $\checkmark$ \\
\hline [Yoon et al. 2007] & $\checkmark$ & $\mathrm{k}-\mathrm{NN}$ & $\checkmark$ & $\checkmark$ & - & - & $\checkmark$ \\
\hline [Jong et al. 2007] & $\checkmark$ & Data Mining & - & $\checkmark$ & - & - & $\checkmark$ \\
\hline [Chen et al. 2007b] & $\checkmark$ & Clustering & $\checkmark$ & $\checkmark$ & - & - & $\checkmark$ \\
\hline
\end{tabular}

O estudo apresentado em [Chen et al. 2007a] utiliza Regras de Associação para o diagnóstico de mal entendimento do conteúdo durante a aprendizagem. O trabalho busca identificar regras comuns em conceitos mal entendidos pelos aprendizes para poder diagnosticar e remediar os aprendizes que são enquadrados pelas regras encontradas.

O artigo [Yoon et al. 2007] aplica o método k-Nearest Neighbor (k-NN) para diagnosticar o Estilo de Aprendizagem dos aprendizes com base no conteúdo ensinado. Tal método utiliza a Distância Euclidiana para classificar um ponto em um plano cartesiano a partir de determinado número de vizinhos mais próximos.

O trabalho [Jong et al. 2007] apresenta um sistema para diagnóstico do comportamento dos aprendizes a partir de dados de portfólio. Técnicas de mineração de dados (Data Mining) foram utilizadas. O experimento foi conduzido para verificar o desempenho do sistema no que diz respeito à capacidade de melhora do resultado acadêmico dos aprendizes através do envio de mensagens baseado na classificação do comportamento.

Em [Chen et al. 2007b], os autores propõem um modelo para realizar o diagnóstico do mal entendimento sobre o conteúdo dos aprendizes com base em dados de portfólio. A técnica utilizada para realizar o diagnóstico foi Clusterização (Clustering), buscando agrupar aprendizes com portfólio semelhante.

Uma comparação dos trabalhos é apresentada na Tabela 1, onde foi indicada a presença $(\checkmark)$ ou ausência ( - ) dos critérios analisados, com exceção da técnica utilizada, que é descritiva. Os critérios utilizados para comparar os trabalhos são os seguintes: Diagnóstico (D): indica se o trabalho realiza o diagnóstico dos aprendizes; Técnica (T): apresenta qual técnica de diagnóstico foi utilizada em cada um dos trabalhos; Educação a Distância (EaD): identifica se o trabalho foca na modalidade de EaD; Dados Históricos (DH): indica se dados históricos são utilizados para diagnosticar as necessidades dos aprendizes; Estilo de Aprendizagem (EA): indica se o trabalho considera estilos de aprendizagem; Trilha de Aprendizagem (TA): este critério evidencia se o trabalho utiliza o conceito de trilhas para estruturar os dados históricos no âmbito de sistemas para educação; Flexibilidade (F): objetiva identificar se o trabalho pode ser expandido ou se permite adicionar, trocar ou remover variáveis no sistema sem reprogramação, de maneira a proporcionar dinamicidade.

Como pode ser visto na Tabela 1 , as técnicas de diagnóstico se mostram variadas não se repetindo para nenhum trabalho. O critério EaD é apresentado pela maioria 
dos trabalhos, assim como os dados históricos e a flexibilidade. O Estilo de Aprendizagem, critério fundamental para este trabalho, não é atendido por nenhum dos trabalhos relacionados, também, nota-se que nenhum trabalho usou o conceito de Trilhas de Aprendizagem.

\section{Modelo Proposto}

Esste artigo propõe um método denominado CPAD (Coletar, Preprocessar, Analisar e Diagnosticar), o modelo coleta dados brutos de sistemas externos, processa esses dados em Trilhas de Aprendizagem(TA), analisa as TAs e realiza o diagnóstico dos aprendizes com base apenas no EA individual.

A arquitetura do modelo é organizada em quatro componentes (Figura 1). Sendo dois internos (Aplicação e CPAD) que implementam as funcionalidades propostas e dois externos (AVA e Questionário) que disponibilizam os dados utilizados pelo modelo. A Aplicação é responsável pela interface, enquanto o CPAD implementa as funcionalidades propostas. O AVA tem como função prover os dados históricos dos aprendizes, já o Questionário representa o componente de coleta da preferência de aprendizagem dos aprendizes através de um questionário que implementa o ILSQ [Felder and Soloman 2013].

O CPAD é o principal componente do modelo, formado por quatro subcomponentes (Coleta, Pré-processamento, Análise e Diagnóstico). Sua estrutura é orientada a agentes [Jennings 2001], onde cada subcomponente é gerenciado por um agente. As rotinas implementadas pelos subcomponentes do CPAD estão identificadas por letras na Figura 1, assim como os seus passos que são identificados por números.

A rotina de coleta $(\mathrm{C})$ obtém os dados brutos e os armazena no Banco de Dados Bruto. Essa rotina é manipulada pelo Agente de Coleta que mantém os dados atualizados e informa o Agente de Pré-processamento indicando que há novos dados para serem processados. A rotina de pré-processamento $(\mathrm{P})$, organiza e estrutura os dados brutos em

Figura 1. Arquitetura do Modelo

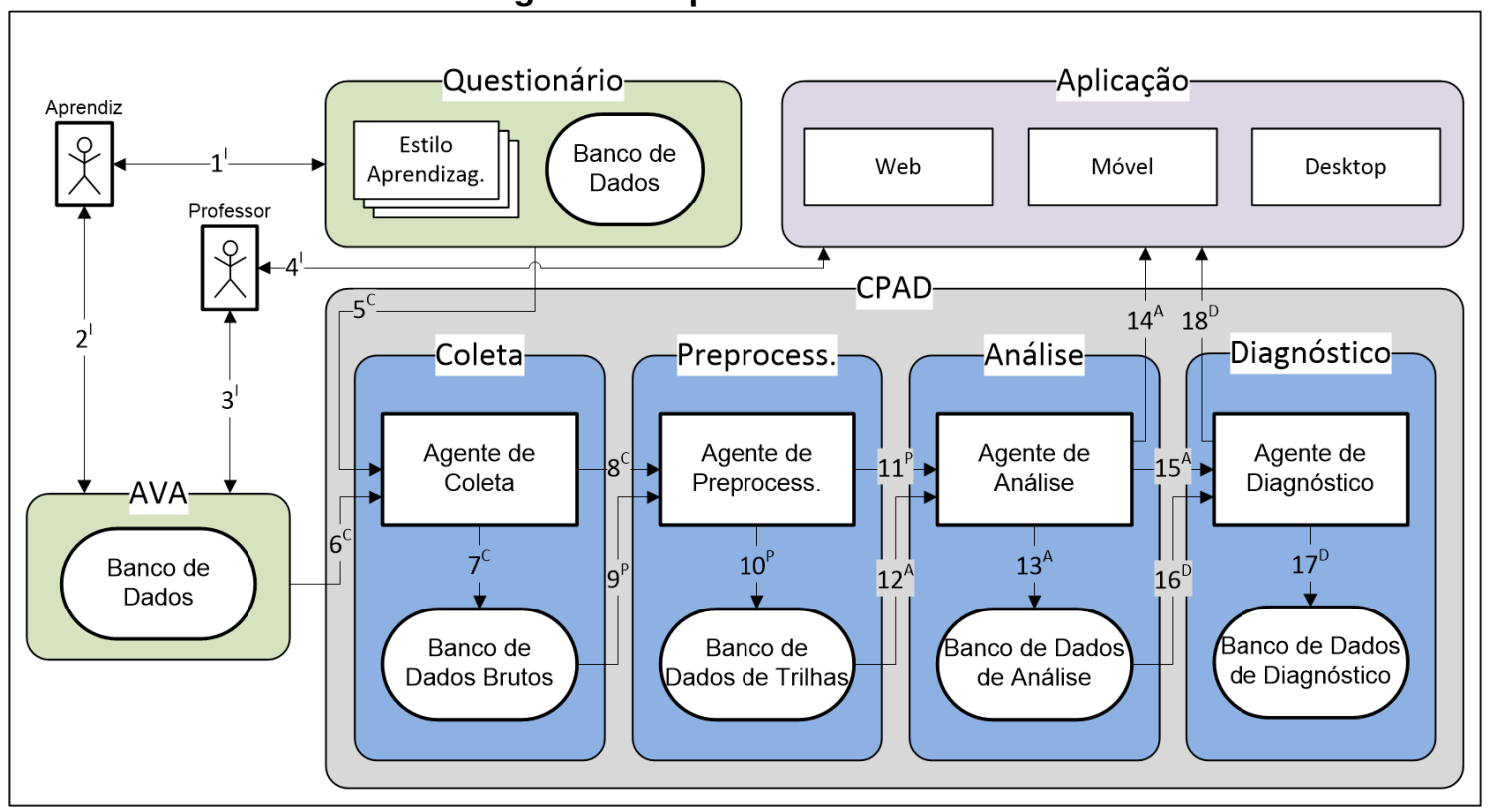


TAs para que possam ser utilizados na etapa de análise. A rotina de análise (A) avalia os critérios utilizados para detectar trilhas dos aprendizes que possam ser consideradas relevantes, esses critérios podem ser manipulados de acordo com a necessidade. Por fim, a rotina de diagnóstico (D) realiza o diagnóstico dos aprendizes considerando o Estilo de Aprendizagem. O processo completo de funcionamento envolve dezoito passos. Cada passo representa a interação entre dois elementos do modelo.

1. Aprendiz e Questionário: Neste passo, o aprendiz acessa o Questionário para responder o questionário de EA. Todas as respostas são mantidas no Banco de Dados. Esta interação possui o fluxo de dados nos dois sentidos;

2. Aprendiz e AVA: Quando o aprendiz acessa o AVA, todos os seus dados de log são salvos no Banco de Dados, como mensagens trocadas no fórum de discussão ou realização de tarefas agendadas pelo professor. Este passo também possui fluxo de dados nos dois sentidos;

3. Professor e AVA: é o momento em que o professor acessa o AVA para agendar uma tarefa, responder à uma pergunta de um aprendiz ou mesmo participar do fórum. Novamente a fluxo de dados ocorre em dois sentidos;

4. Professor e Aplicação: Este passo remete à utilização da Aplicação. As funcionalidades de análise e diagnóstico são acessadas pelo professor através da uma interface. É através desta interface que o professor consegue analisar padrões de necessidades dos aprendizes e diagnosticá-los, podendo assim tomar uma decisão embasada nos resultados obtidos. Este é o último passo que possui dois sentidos em seu fluxo de dados;

5. Questionário e Agente de Coleta: Neste passo o Agente de Coleta monitora a disponibilidade de novos dados de estilo dos aprendizes. No momento em que há dados disponíveis, o Agente de Coleta inicia a requisição das informações buscando manter o Banco de Dados Bruto atualizado. Este passo inicia a rotina de sincronização dos estilos que ocorre entre o Banco de Dados do Questionário e Banco de Dados Bruto;

6. AVA e Agente de Coleta: Este passo inicia a rotina de sincronização dos dados de $\log$ do AVA. O monitoramento executado pelo Agente de Coleta busca atualizar o Banco de Dados Bruto com dados provenientes do Banco de Dados do AVA;

7. Agente de Coleta e Banco de Dados Bruto: Este corresponde ao segundo passo na sincronização dos dados, tanto os de estilos (passo 5) quanto os de log (passo 6). É neste passo que os dados são efetivamente salvos pelo Agente de Coleta no Banco de Dados Bruto.

8. Agente de Coleta e Agente de Pré-processamento: Quando ambos os processos de sincronização são finalizados, um relatório é enviado ao Agente de Préprocessamento informando que a rotina de pré-processamento pode ser inicializada;

9. Banco de Dados Bruto e Agente de Pré-processamento: As informações do Banco de Dados Bruto podem agora ser acessadas objetivando a estruturação dessas informações em TAs;

10. Agente de Pré-processamento e Banco de Dados de Trilhas: Uma vez estruturadas, as informações são salvas no banco de dados de trilhas. Essas informações tornam possíveis as rotinas de análise e diagnóstico;

11. Agente de Pré-processamento e Agente de Análise: Este passo corresponde ao 
envio do relatório indicando que há trilhas disponíveis e que a rotina de análise pode ser inicializada;

12. Banco de Dados de Trilhas e Agente de Análise: Neste passo as trilhas armazenadas são requisitadas para serem analisadas com o objetivo de identificar as trilhas relevantes de acordo com os critérios definidos. Em todas as análises são indispensáveis tanto os dados de trilhas quanto os dados de estilos;

13. Agente de Análise e Banco de Dados de Análise: Uma vez que a análise é executada, os resultados são salvos no Banco de Dados de Análise. A análise pode ser executada manual ou automaticamente;

14. Agente de Análise e Aplicação: Este passo ocorre no momento em que o professor acessa a interface para executar uma análise, um diagnóstico ou mesmo visualizar resultados anteriores;

15. Agente de Análise e Agente de Diagnóstico: Após a execução da análise, o Agente de Diagnóstico recebe um relatório informando que a rotina de diagnóstico pode ser executada;

16. Banco de Dados de Análise e Agente de Diagnóstico: Quando um diagnóstico é realizado, as informações de análise são requisitadas. Cada diagnóstico pode utilizar como base uma única análise;

17. Agente de Diagnóstico e Banco de Dados de Diagnóstico: Neste passo o agente de diagnóstico, após finalizar sua rotina, salva os resultados obtidos no banco de dados de diagnóstico;

18. Agente de Diagnóstico e Aplicação: Ocorre quando um professor executa um diagnóstico através da interface ou solicita a visualização de um anterior.

\section{Aspectos de Implementação}

Todos os componentes foram implementados, com excessão do AVA. Como AVA está sendo usado o Moodle. Um questionário baseado no ILSQ de [Felder and Soloman 2013] e traduzido por [Kuri and Giorgetti 1996] foi utilizado para a coleta do Estilo de Aprendizagem através de uma aplicação $w e b$, que foi disponibilizada aos aprendizes dos cursos de EaD da UNISINOS. Esta aplicação corresponde ao componente Questionário. O subcomponente Aplicação fornece uma interface para as funcionalidades oferecidas pela CPAD. Complementarmente, a Aplicação dispõe de visualizações dos dados e de gráficos das análises e diagnósticos. As linguagens utilizadas foram Visual C\#, ASP.net e o banco de dados SQL Server 2012.

\section{Aspectos de Avaliação}

Os experimentos buscaram avaliar a relevância do Estilo de Aprendizagem para diagnosticar antecipadamente o comportamento de aprendizes. Para isso foram coletados os Estilos de Aprendizagem de 480 alunos das disciplinas de EaD na UNISINOS (Figura 2). Dentre essas, foram escolhidas as quatro que apresentaram o maior número de alunos que responderam ao questionário (Tabela 2), consequentemente a amostra foi composta por 202 estudantes. A Tabela 2 mostra o total de alunos em cada disciplina, sendo que um dos alunos da mostra pode estar matriculado em mais de uma disciplina.

Os cenários avaliados para cada uma das disciplinas são compostos pelas dimensões do Estilo de Aprendizagem e suas possíveis combinações (Tabela 3). O comportamento escolhido para a avaliação foi a Evasão. No experimento foi considerado 
Tabela 2. Disciplinas Avaliadas

\begin{tabular}{lc}
\hline \hline Disciplina & Quantidade de Aprendizes \\
\hline \hline Laboratório I & 98 \\
Lógica & 123 \\
Matemática & 95 \\
Programação I & 100 \\
\hline \hline
\end{tabular}

Tabela 3. Cenários Combinando as Dimensões do Estilo de Aprendizagem

\begin{tabular}{cccccl}
\hline \hline Cenário & A/R & S/I & V/V & S/G & Descrição \\
\hline \hline 1 & $\checkmark$ & - & - & - & Ativo/Reflexivo \\
2 & - & $\checkmark$ & - & - & Sensitivo/Intuitivo \\
3 & - & - & $\checkmark$ & - & Visual/Verbal \\
4 & - & - & - & $\checkmark$ & Sequencial/Global \\
5 & $\checkmark$ & $\checkmark$ & - & - & Ativo/Reflexivo, Sensitivo/Intuitivo \\
6 & $\checkmark$ & - & $\checkmark$ & - & Ativo/Reflexivo, Visual/Verbal \\
7 & $\checkmark$ & - & - & $\checkmark$ & Ativo/Reflexivo, Sequencial/Global \\
8 & - & $\checkmark$ & $\checkmark$ & - & Sensitivo/Intuitivo, Visual/Verbal \\
9 & - & $\checkmark$ & - & $\checkmark$ & Sensitivo/Intuitivo, Sequencial/Global \\
10 & - & - & $\checkmark$ & $\checkmark$ & Visual/Verbal, Sequencial/Global \\
11 & $\checkmark$ & $\checkmark$ & $\checkmark$ & - & Ativo/Reflexivo, Sensitivo/Intuitivo, Visual/Verbal \\
12 & $\checkmark$ & $\checkmark$ & - & $\checkmark$ & Ativo/Reflexivo, Sensitivo/Intuitivo, Sequencial/Global \\
13 & $\checkmark$ & - & $\checkmark$ & $\checkmark$ & Ativo/Reflexivo, Visual/Verbal, Sequencial/Global \\
14 & - & $\checkmark$ & $\checkmark$ & $\checkmark$ & Sensitivo/Intuitivo, Visual/Verbal, Sequencial/Global \\
15 & $\checkmark$ & $\checkmark$ & $\checkmark$ & $\checkmark$ & Ativo/Reflexivo, Sensitivo/Intuitivo, Visual/Verbal, Sequencial/Global \\
\hline \hline
\end{tabular}

que o aluno evadiu quando ele não participou de um dos dois exames finais da disciplina. Foram escolhidas quatro diferentes técnicas de diagnóstico, de modo a oferecer uma comparação entre os cenários. As técnicas selecionadas foram a árvore de Decisão (DT), o Naïve Bayes (NB), a Rede Neural Artificial (RNA) e o Support Vector Machine (SVM). Estas técnicas têm sido utilizadas para classificar aprendizes em vários trabalhos [Liao et al. 2012, Manghirmalani et al. 2011]. A discussão dos resultados considerou as médias dos valores encontrados através das diferentes técnicas.

Os principais resultados são apresentados na Tabela 4. Nota-se que cada disciplina apresentou um diferente cenário com a melhor precisão média. Esses cenários apresentam apenas uma dimensão de estilo de aprendizagem, evidenciando que não há apenas um

Figura 2. Caracterização da Amostra
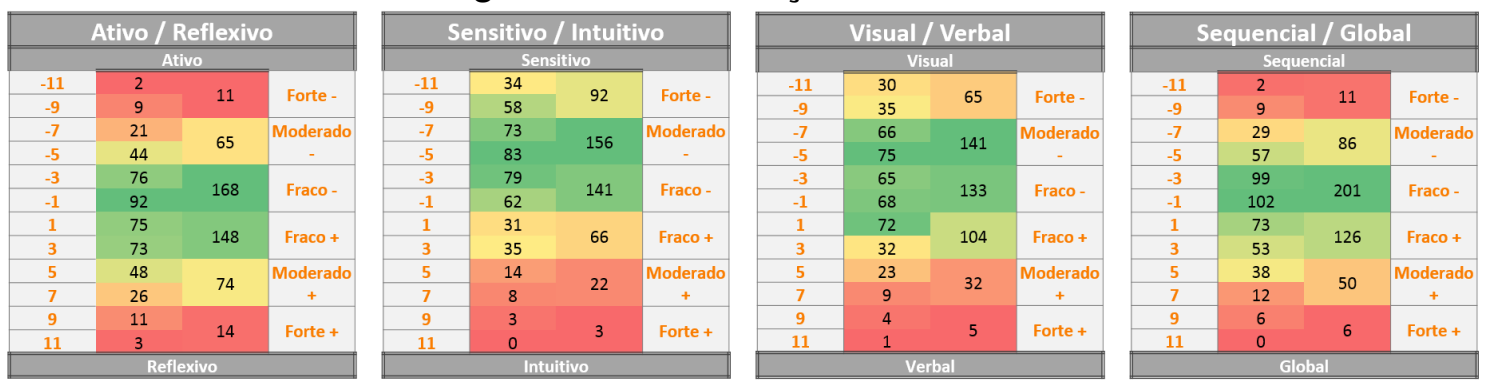
Tabela 4. Experimentos Realizados e suas Principais Características

\begin{tabular}{|c|c|c|c|c|c|c|c|c|}
\hline \multirow{2}{*}{ Disciplina } & \multicolumn{2}{|c|}{ Melhor Média } & \multicolumn{2}{|c|}{ Pior Média } & \multicolumn{2}{|c|}{ Melhor Desvio Padãro } & \multicolumn{2}{|c|}{ Pior Desvio Padrão } \\
\hline & Cenário & $\mathrm{M}(\%)$ & Cenário & $\mathrm{M}(\%)$ & Cenário & $\mathrm{DP}(\%)$ & Cenário & $\mathrm{DP}(\%)$ \\
\hline Laboratório I & 2 & 68.33 & 15 & 64.11 & 4 & 0.60 & 6 & 4.80 \\
\hline Lógica & 4 & 79.74 & 5 & 78.32 & 4 & 0.00 & 15 & 2.63 \\
\hline Matemática & 1 & 68.88 & 8 & 62.22 & 9 & 0.62 & 15 & 8.93 \\
\hline Programação I & 3 & 70.00 & 15 & 67.17 & 3 & 0.00 & 8 & 4.00 \\
\hline
\end{tabular}

estilo que pode explicar o comportamento de evasão. Mesmo obtendo valores próximos com outros cenários, não pode-se definir uma dimensão como sendo destacada. No entanto, o resultado sugere que é possível ter um cenário apropriado, dependendo do caso.

A menor precisão média foi encontrada em cenários com mais de uma dimensão de aprendizagem. Em todos os piores casos existe a presença da dimensão Sensitivo/Intuitivo e em dois casos observa-se o cenário 15.

O cenário 15 também esteve presente em dois casos onde o desvio padrão era mais elevado. A dimensão Sequencial/Global esteve presente nos três cenários com o melhor desvio padrão. Adicionalmente, quando a dimensão Sequencial/Global esteve presente, os valores médios estavam entre os mais altos e o desvio padrão entre os mais baixos.

A dimensão do estilo Sequencial/Global considera como um estudante compreende e recebe a informação no contexto de aprendizagem [Felder and Silverman 1988]. Segundo [Schmeck 1988], Sequencial/Global é a principal dimensão cognitiva. Analisando essa dimensão pode-se notar uma diferença entre os alunos que evadiram e os que não evadiram.

Entre os alunos que não evadiram, o índice de estilo de aprendizagem era Sequencial Fraco (Figura 2). Aprendizes sequenciais são analíticos, possuem um processo de pensamento linear e aprendem em pequenos passos sequenciais [Riding and Sadler-Smith 1997]. Entre os estudantes que evadiram o índice de preferência do estilo Sequencial/Global estava entre 1 e 3. O que mostra que um pequeno grupo de aprendizes possuía uma ligeira preferência por um processo de aprendizagem global.

Podemos entender que os aprendizes com preferência por um processo de aprendizado global, mesmo que fraco, não receberam o suporte apropriado de acordo com suas preferências de aprendizagem. Como exemplo pode-se citar materiais, métodos de ensino ou atividades que fossem capazes de atender suas preferências globais. Isso pode ser explicado pela natureza das disciplinas EaD. Todas as disciplinas analisadas são compostas de nove módulos, um por semana e cada módulo contém um conteúdo específico. Para cada semana os aprendizes necessitam concentrar-se no conteúdo semanal e não preocuparem-se com o que será apresentado posteriormente. Essa abordagem tende a favorecer aprendizes sequenciais. Considerando-se a mostra analisada, pode-se concluir que para a amostra analisada, as preferências de aprendizado são significantemente diferentes e poderiam explicar o comportamento de evasão. 


\section{Conclusões}

Este artigo propôs um modelo para utilização do Estilo de Aprendizagem como variável no diagnóstico antecipado do comportamento dos aprendizes da Educação a Distância. Diferentemente dos trabalhos relacionados, o modelo proposto realiza a tarefa de diagnosticar o comportamento utilizando o Estilo de Aprendizagem dos aprendizes. A utilização de Trilha de Aprendizagem também é um diferencial deste trabalho. Este conceito ajuda a entender a sequência de interações dos aprendizes e permite filtrar as informações de contexto para avaliação do seu comportamento.

O protótipo foi aplicado em um experimento focando no diagnóstico da evasão. Os resultados permitiram constatar a potencial utilização do Estilo de aprendizagem para antecipar o comportamento dos aprendizes em diferentes disciplinas da EaD.

Entre as frentes que podem ser exploradas como trabalhos futuros está o mapeamento do Estilo de Aprendizagem para Objetos de Aprendizagem. Isso permitiria identificar se o conteúdo de uma determinada disciplina está preparado para suportar todos os aprendizes e suas diferentes preferências. Ainda, análises de desempenho acadêmico poderiam ser realizadas buscando verificar a eficácia de materiais direcionados a estilos de aprendizagem.

A aplicação das análises feitas neste trabalho focando no ensino presencial também seria outra questão de pesquisa. Complementarmente, poderia ser feito o mapeamento do Estilo de Aprendizagem dos Professores e analisar o quanto o educador pode potencializar o aprendizado quando seu estilo é similar ao do aprendiz. Este estudo permitiria análises prévias para a alocação de professores.

\section{Agradecimentos}

Os autores agradecem ao CNPq e a empresa GVDASA pelo suporte à realização desse trabalho.

\section{Referências}

Chen, C.-M., Hsieh, Y.-L., and Hsu, S.-H. (2007a). Mining learner profile utilizing association rule for web-based learning diagnosis. Expert Systems with Applications, 33(1):6-22.

Chen, C.-M., Li, C.-Y., Chan, T.-Y., Jong, B.-S., and Lin, T.-W. (2007b). Diagnosis of students online learning portfolios. Frontiers In Education Conference - Global Engineering: Knowledge Without Borders, Opportunities Without Passports (FIE 2007), 37th Annual, pages $17-22$.

Chen, L.-H. (2011). Enhancement of student learning performance using personalized diagnosis and remedial learning system. Computers \& Education, 56(1):289 - 299.

Chen, S.-M. and Bai, S.-M. (2009). Learning barriers diagnosis based on fuzzy rules for adaptive learning systems. Expert Systems with Applications, 36(8):11211 - 1122.

Dey, A. K., Abowd, G. D., and Salber, D. (2001). A conceptual framework and a toolkit for supporting the rapid prototyping of context-aware applications. Human-Computer Interaction, 16(2):97 - 166. 
Felder, R. M. and Silverman, L. (1988). Learning and teaching styles in engineering education. Engineering Education, 78(June):674 - 681.

Felder, R. M. and Soloman, B. (2013). Index of learning styles questionnaire. http://www.engr.ncsu.edu/learningstyles/ilsweb.html. Acessed in 26/04/2013.

Jennings, N. R. (2001). An agent-based approach for building complex software systems. Communications of the ACM, 44(4):35-41.

Johnson, G. and Johnson, J. (2010). Dimensions of online behavior: Implications for engineering e-learning. In Iskander, M., Kapila, V., and Karim, M. A., editors, Technological Developments in Education and Automation, pages 61-66. Springer Netherlands.

Jong, B.-S., Chan, T.-Y., and Wu, Y.-L. (2007). Learning log explorer in e-learning diagnosis. IEEE Transactions on Education, 50(3):216 - 228.

Kuri, N. P. and Giorgetti, M. F. (1996). Índice de estilos de aprendizagem (index of learning styles). São Paulo: Escola de Engenharia de São Carlos.

Lee, C.-H., Lee, G.-G., and Leu, Y. (2009). Application of automatically constructed concept map of learning to conceptual diagnosis of e-learning. Expert Systems with Applications, 36(2, Part 1):1675 - 1684.

Liao, S.-H., Chu, P.-H., and Hsiao, P.-Y. (2012). Data mining techniques and applications a decade review from 2000 to 2011. Expert Systems with Applications, 39(12):11303 $-11311$.

Manghirmalani, P., Panthaky, Z., and Jain, K. (2011). Learning disability diagnosis and classification - a soft computing approach. IEEE World Congress on Information and Communication Technologies (WICT 2011), pages 479 - 484.

Moore, M. (1993). Theory of transactional distance. Routledge, Oxon.

Peterson, E. R., Rayner, S. G., and Armstrong, S. J. (2009). Researching the psychology of cognitive style and learning style: Is there really a future? Learning and Individual Differences, 19(4):518 - 523.

Riding, R. J. and Sadler-Smith, E. (1997). Cognitive Style and Learning Strategies: Some Implications for Training Design. International Journal of Training and Development, 1(3):199 - 208.

Schmeck, R. R. (1988). Learning Strategies and Learning Styles, page 368. Plenum.

Siemens, G. and Long, P. (2011). Penetrating the fog: Analytics in learning and education. Educause Review, 46(5):30-32.

Silva, J., Rosa, J., Barbosa, J., Barbosa, D., and Palazzo, L. (2010). Content distribution in trail-aware environments. Journal of the Brazilian Computer Society, 16(3):163 176.

$\mathrm{Wu}, \mathrm{Y}$. (2012). Adaptive learning diagnosis mechanisms for e-learning. Lecture Notes in Computer Science, 7196(Ldd):102 - 110.

Yoon, T.-B., Choi, M. A., Wang, E., Lee, J. H., and Kim, Y. S. (2007). Improvement of learning styles diagnosis based on outliers reduction of user interface behaviors. Ninth IEEE International Symposium on Multimedia Workshops (ISMW 2007), pages 497 503. 